\title{
Stable, Strongly Attractive, Two-State Mixture of Lithium Fermions in an Optical Trap
}

\author{
K. M. O'Hara, M. E. Gehm, S. R. Granade, S. Bali, and J. E. Thomas \\ Physics Department, Duke University, Durham, North Carolina 27708-0305
}

(November 18, 2018)

\begin{abstract}
We use an all-optical trap to confine a strongly attractive two-state mixture of lithium fermions. By measuring the rate of evaporation from the trap, we determine the effective elastic scattering cross section $4 \pi a^{2}$ to show that the magnitude of the scattering length $|a|$ is very large, in agreement with predictions. We show that the mixture is stable against inelastic decay provided that a small bias magnetic field is applied. For this system, the s-wave interaction is widely tunable at low magnetic field, and can be turned on and off rapidly via a Raman $\pi$ pulse. Hence, this mixture is well suited for fundamental studies of an interacting Fermi gas.
\end{abstract}

PACS numbers: 32.80.Pj

Copyright 2000 by the American Physical Society

Trapped, ultracold atomic vapors offer exciting new opportunities for fundamental studies of an interacting Fermi gas in which the temperature, density and interaction strength can be independently controlled. Recently, a degenerate gas of fermionic ${ }^{40} \mathrm{~K}$ has been produced by using a two-state mixture to enable s-wave scattering and evaporation in a magnetic trap [1]. By removing one species, the properties of the noninteracting degenerate gas were measured, demonstrating that the momentum distribution and the total energy obey Fermi-Dirac statistics [1]. However, the properties of interacting two-state fermionic vapors have not been explored experimentally.

Theoretical treatments of an interacting Fermi gas have focused extensively on ${ }^{6} \mathrm{Li}$ [2 9]. Certain twostate ${ }^{6} \mathrm{Li}$ mixtures are predicted to be strongly attractive, i.e., they have anomalously large and negative scattering lengths 10] arising from a near-zero energy resonance in the triplet state [11]. It has been predicted that these strongly attractive mixtures can undergo a transition to a superfluid state at a relatively high transition temperature [2, [n]. In addition, the two-state effective interaction potential is widely tunable in a magnetic field, permitting systematic studies of fundamental phenomena such as collective oscillations for both the normal and superfluid phases [3,5],6], as well as new tests of superconductivity theory

Unfortunately, magnetically trappable mixtures in ${ }^{6} \mathrm{Li}$ with large s-wave scattering lengths are not stable, since there are correspondingly large spin-exchange and dipolar decay rates [2,7, 10]. Hence, the methods employed to study degenerate ${ }^{40} \mathrm{~K}$ are not applicable. For this reason, we developed an ultrastable $\mathrm{CO}_{2}$ laser trap to confine a stable mixture of the two lowest ${ }^{6} \mathrm{Li}$ hyperfine states $[12$. However, attaining a large and negative scattering length in this mixture requires high magnetic fields $B \geq 800 \mathrm{G}$ to exploit either a Feshbach resonance or the triplet scattering length [0,10].

In this Letter, we show that there exists another stable hyperfine state mixture in ${ }^{6} \mathrm{Li}$ which has the following unique properties. First, we predict that the scattering length $a$ is large, negative, and widely tunable at low magnetic field $B$. By monitoring the rate of evaporation from the $\mathrm{CO}_{2}$ laser trap at a fixed well depth, we measure $|a|=540_{-100}^{+210} a_{0}$ at $B=8.3 \mathrm{G}$. This result confirms for the first time that very large scattering lengths exist in ${ }^{6} \mathrm{Li}$ mixtures. The predicted scattering length is $-490 a_{0}$ at $B=8.3 \mathrm{G}$, consistent with our observations, and is expected to increase to $-1615 a_{0}$ as $B \rightarrow 0$. Second, we find that this system is stable against spin exchange collisions provided that $B \neq 0$. In addition, the dipolar decay rate is predicted to be very small [13], consistent with our observations. Finally, in the experiments, a Raman $\pi$ pulse is employed to abruptly create an interacting mixture from a noninteracting one, a desirable feature for studies of many-body quantum dynamics [14].

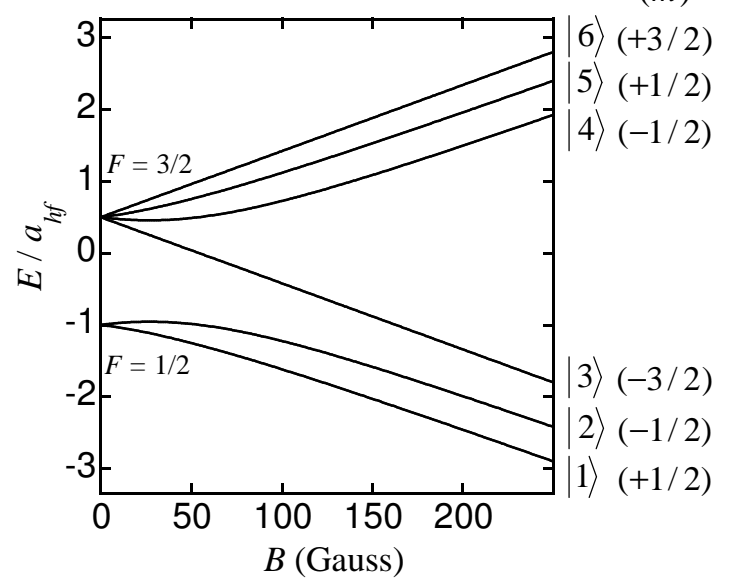

FIG. 1. ${ }^{6} \mathrm{Li}$ hyperfine states, labeled $|1\rangle$ to $|6\rangle$ in order of increasing energy in a magnetic field. The magnetic quantum number of each state is denoted by $m$. The hyperfine constant $a_{h f}=152.1 \mathrm{MHz}$.

Fig. 11 shows the hyperfine states for ${ }^{6} \mathrm{Li}$ labelled $|1\rangle-$ $|6\rangle$, in order of increasing energy in a magnetic field. At low field, the states $|1\rangle$ and $|2\rangle$ correspond to the $\mid F=$ $1 / 2, m\rangle$ states, while states $|3\rangle$ through $|6\rangle$ correspond to states $|F=3 / 2, m\rangle$. At nonzero magnetic field, only the magnetic quantum number $m$ is conserved. The subject of this paper is the $|3\rangle-|1\rangle$ mixture. 


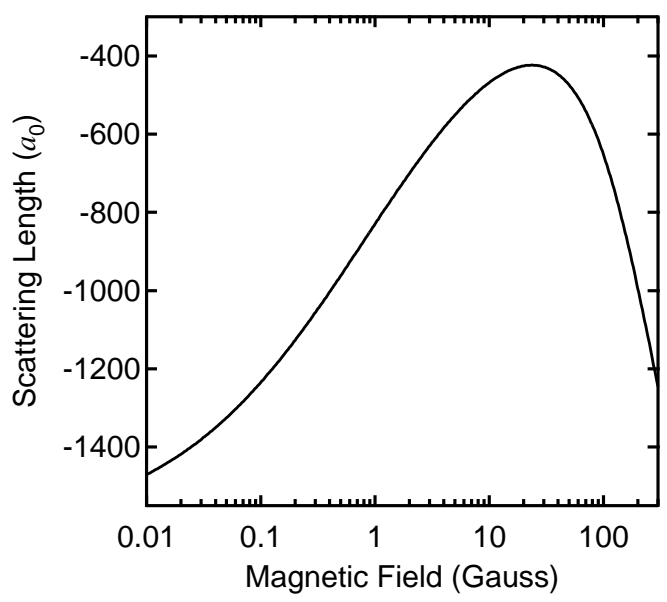

FIG. 2. Magnetic field dependence of the scattering length $a_{31}$ for a mixture of the $|3\rangle$ and $|1\rangle$ hyperfine states of ${ }^{6} \mathrm{Li}$.

Fig. 2 2 shows the scattering length $a_{31}$ for the $|3\rangle-|1\rangle$ mixture as a function of magnetic bias field $B$. We estimate $a_{31}(B)$ by using the asymptotic boundary condition $(\mathrm{ABC})$ approximation [10]. This calculation incorporates the singlet and triplet scattering lengths [11, $a_{S}=45.5 \pm 2.5 a_{0}$ and $a_{T}=-2160 \pm 250 a_{0}$ and a boundary radius which we take to be $R=40 a_{0}$ 10. The scattering length varies from $-1620 a_{0}\left(\simeq 3 a_{T} / 4\right.$ as $\left.B \rightarrow 0\right)$ to $-480 a_{0}$ at $B=10 \mathrm{G}$. The results of our approximate calculation for $B=0$ to $B=200 \mathrm{G}$ are confirmed within $10 \%$ by van Abeelen and Verhaar [13] using a coupled channel calculation which includes the uncertainties in the potentials. At higher fields, near $800 \mathrm{G}$, we believe the scattering length exhibits a Feshbach resonance (not shown). Above this resonance, the scattering length approaches the triplet scattering length of $-2160 a_{0}$.

The $|3\rangle-|1\rangle$ mixture is stable against spin-exchange collisions provided that a small bias magnetic field is applied. Spin-exchange inelastic collisions conserve the two-particle total magnetic quantum number $M_{T}$, where $M_{T}=-1$ for the $|\{3,1\}\rangle$ state. Note that $\{$,$\} denotes$ the antisymmetric two-particle spin state, as required for s-wave scattering which dominates at low temperatures. There are no lower-lying antisymmetric states with $M_{T}=-1$. Hence, exothermic collisions are precluded. The only other states with $M_{T}=-1$ are $|\{4,2\}\rangle$ and $|\{5,3\}\rangle$. Without an adequate bias magnetic field, transitions to these states lead to population in level $|4\rangle$. Then, exothermic $|\{3,4\}\rangle \rightarrow|\{3,2\}\rangle$ and $|\{4,1\}\rangle \rightarrow|\{1,2\}\rangle$ collisions can occur. With an adequate bias magnetic field, the energy of states $|\{4,2\}\rangle$ and $|\{5,3\}\rangle$ can be increased relative to that of state $|\{3,1\}\rangle$ by more than the maximum relative kinetic energy, i.e., twice the well depth during evaporative cooling. By energy conservation, spin-exchange transfer is then suppressed. In this case, the inelastic rate is limited to magnetic dipoledipole (dipolar) interactions which contain a rank 2 relative coordinate operator of even parity [2]. Since parity is conserved and $\mathrm{p}$ - wave $\rightarrow \mathrm{p}$ - wave scattering is frozen out at low temperature, the dominant dipolar process is a small $\mathrm{s} \rightarrow \mathrm{d}$ rate in which $|\{3,1\}\rangle \rightarrow|\{1,2\}\rangle$ 13].

In the experiments, the $\mathrm{CO}_{2}$ laser trap is initially loaded from a magneto-optical trap (MOT) 12]. At the end of the loading period, the MOT laser beams are tuned near resonance and the intensity is lowered to decrease the temperature. Then, optical pumping is used to empty the $F=3 / 2$ state to produce a 50-50 mixture of the $|1\rangle-|2\rangle$ states. These states are noninteracting at low magnetic field, i.e, the scattering amplitude vanishes as a result of an accidental cancellation 10. With a $\mathrm{CO}_{2}$ laser trap depth of $330 \mu \mathrm{K}$, up to $4 \times 10^{5}$ atoms are confined in the lowest-lying hyperfine states at an initial temperature between 100 and $200 \mu \mathrm{K}$. A bias magnetic field of $8.3 \mathrm{G}$ is applied to split the two-particle energy states by $\simeq 16 \mathrm{MHz}$. This is twice the maximum attainable energy at our largest well depth of $400 \mu \mathrm{K}=8 \mathrm{MHz}$. After a delay of 0.5 second relative to the loading phase, a pair of optical fields is pulsed on to induce a Raman $\pi$ pulse. This pulse transfers the population in state $|2\rangle$ to state $|3\rangle$ in two microseconds, initiating evaporative cooling in the resulting $|3\rangle-|1\rangle$ mixture. The optical fields are detuned from resonance with the D2 transition by $\simeq 700 \mathrm{MHz}$ to suppress optical pumping. If the Raman pulse is not applied, the trapped atoms remain in the noninteracting $|1\rangle-|2\rangle$ mixture and exhibit a purely exponential decay with a time constant $\simeq 300$ seconds.

An acousto-optic modulator $(\mathrm{A} / \mathrm{O})$ in front of the $\mathrm{CO}_{2}$ laser controls the laser intensity, which is reduced to yield a shallow trap depth of $100 \mu \mathrm{K}$. By using a shallow well, we avoid the problem that the elastic cross section becomes independent of the scattering length at high energy, as described below. In addition, the shallow well greatly reduces the number of loaded atoms and makes the sample optically thin, simplifying calibration of the number of trapped atoms. To determine the trap parameters, the laser power is modulated and parametric resonances [15] are observed at drive frequencies of $2 \nu$ for three different trap oscillation frequencies $\nu$ : At $100 \mu \mathrm{K}$ well depth, $\nu_{x}=2.4 \mathrm{kHz} \nu_{y}=1.8 \mathrm{kHz}$ and $\nu_{z}=100 \mathrm{~Hz}$, where the trap laser beam propagates along $\mathbf{z}$. Using the measured total power as a constraint, we obtain the trap intensity $1 / e^{2}$ radii, $w_{x}=50 \mu \mathrm{m}$ and $w_{y}=67 \mu \mathrm{m}$, and the axial intensity $1 / e^{2}$ length, $z_{f} \simeq 1.13 \mathrm{~mm}$, where $z_{f}$ is consistent with the expected Rayleigh length within $15 \%$.

The number of atoms in the trap $N(t)$ is estimated using a calibrated photomultiplier. The detection system monitors the fluorescence induced by pulsed, retroreflected, $\sigma_{ \pm}$probe and repumper beams which are strongly saturating $\left(I / I_{\text {sat }}=26\right.$ for the strongest transition). To simplify calibration, only the isotropic component of the fluorescence angular distribution is measured: The collecting lens is placed at the magic angle [16] of $55^{\circ}$ $\left(P_{2}(\cos \theta)=0\right)$ with respect to the propagation direction of the probe beams. The net efficiency of the detection system is determined using laser light of known power. The primary uncertainty in the calibration arises from 
the excited state population fraction, which we estimate lies between $1 / 4$ and $1 / 2$.

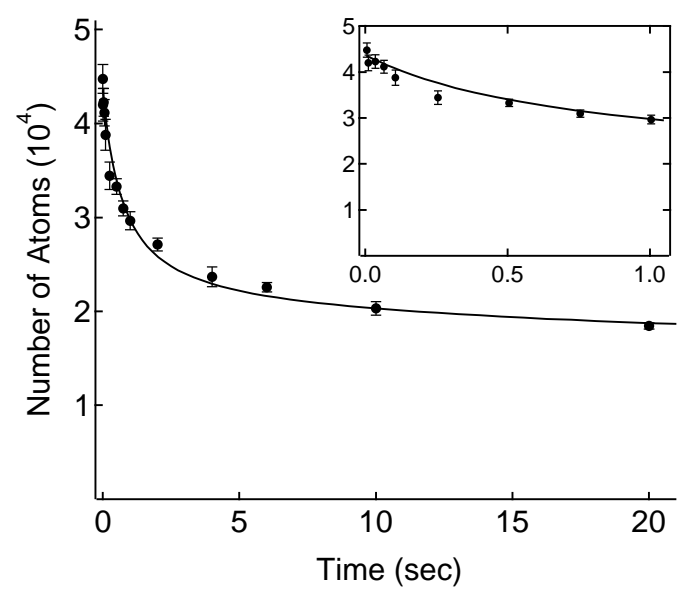

FIG. 3. Number of trapped atoms versus time for evaporation of a $|3\rangle-|1\rangle$ mixture of ${ }^{6} \mathrm{Li}$ at a fixed well depth of $100 \mu \mathrm{K}$. The solid curve shows the s-wave Boltzmann equation fit for a scattering length of $\left|a_{31}\right|=540 a_{0}$. Inset: 0-1 second.

Fig. 3 shows the number of trapped atoms $N(t)$ measured for the $|3\rangle-|1\rangle$ mixture at a well depth $U_{0}=100 \mu \mathrm{K}$ and a bias field of $8.3 \mathrm{G}$ as a function of time between $5 \mathrm{~ms}$ and 20 seconds after evaporation is initiated. For times beyond 50 seconds (not shown), the evaporation stagnates, and we observe an exponential decay of the cooled $|3\rangle-|1\rangle$ mixture with a time constant of 370 seconds over a period of a few hundred seconds. The error bars are the standard deviation of the mean of ten complete runs through the entire time sequence.

A model based on the s-wave Boltzmann equation [17] is used to predict $N(t)$ for comparison to the experiments. This equation is modified to include the density of states for a gaussian potential well [12] and to include the energy dependence of the elastic cross section. Assuming a short range potential and a symmetric (s-wave) spatial state, the cross section takes the form

$$
\sigma(k)=\frac{8 \pi a_{31}^{2}}{1+k^{2} a_{31}^{2}},
$$

where $\hbar k$ is the relative momentum. For $k\left|a_{31}\right|<<1$, the cross section is maximized. When $k\left|a_{31}\right|>>1$, the cross section approaches the unitarity limit $8 \pi / k^{2}$ which is independent of $a_{31}$. Note that $k\left|a_{31}\right|=1$ corresponds to a relative kinetic energy of $\epsilon=\hbar^{2} /\left(2 \mu a_{31}^{2}\right)$, where $\mu=$ $M / 2$ is the reduced mass. For $\left|a_{31}\right|=500 a_{0}, \epsilon=115 \mu \mathrm{K}$.

For a two-state mixture of fermions, the effective cross section is reduced from that of Eq. 1 1 by a factor of 2 since pairs of colliding atoms are in an antisymmetric hyperfine state with a probability $1 / 2$. This effective cross section is used in a Boltzmann collision integral for each state $i=1,3$. A decay term $-N_{i}(t) / \tau$ with $\tau=370 \mathrm{sec}$ is added to account for the measured trap lifetime. A detailed description of our coupled Boltzmann equation model will be published elsewhere.

The coupled s-wave Boltzmann equations for the two states are numerically integrated to determine $N(t)$ using the well parameters as fixed inputs. From the calibrated photomultiplier signal, assuming that $1 / 3$ of the atoms are in the excited state, we obtain an initial total number $N_{0}=44,000$. For this case, the initial collision rate in $\mathrm{Hz}$ is estimated to be $1 /\left(2 \pi \tau_{c}\right) \simeq N_{0} M \sigma_{0} \nu^{3} /\left(k_{B} T\right)$, where $\nu^{3}=\nu_{x} \nu_{y} \nu_{z}, \sigma_{0}=8 \pi a_{31}^{2}$, and $M$ is the ${ }^{6} \mathrm{Li}$ mass. Assuming $\left|a_{31}\right|=500 a_{0}, \tau_{c}=30 \mathrm{~ms}$. Hence, for $t>0.3$ seconds, when on average 10 collisions have occurred, the sample should be thermalized as assumed in the theory.

The best fit to the data starting with 22,000 atoms in each state is shown as the solid curve in Fig. 3. The $\chi^{2}$ per degree of freedom for this fit is 1.4 and is found to be very sensitive to the initial temperature $T_{0}$ of the atoms in the optical trap. ¿From the fit, we find $T_{0}=46 \mu \mathrm{K}$, which is less than the well depth. We believe that this low temperature is a consequence of the MOT gradient magnet, which is turned off after the MOT laser beams. The effective well depth of the optical trap is therefore reduced until the gradient is fully off, allowing hotter atoms to escape before the Raman pulse is applied to create the $|3\rangle-|1\rangle$ mixture. The fit is most sensitive to data for $t>0.5$ second, where the thermal approximation is expected to be valid. From the fit, we obtain the scattering length $\left|a_{31}\right|=540 \pm 25 a_{0}$, which is within $10 \%$ of the predictions of Fig. 2. The quoted error corresponds to a change of 1 in the total $\chi^{2}$.

We determine the systematic errors in $a_{31}$ due to the uncertainties in the calibration and in the population imbalance as follows. The data is fit for an initial number of atoms $N_{0}$ of 58,000 and 29,000, corresponding to an excited state fraction of $1 / 4$ and $1 / 2$. This yields $\left|a_{31}\right|=440 \pm 20 a_{0}$ and $\left|a_{31}\right|=750 \pm 42 a_{0}$, respectively. Note that for the larger scattering lengths, the cross section given by Eq. 11 approaches the unitarity limit and the error increases. We assume that the initial population imbalance for states $|3\rangle$ and $|1\rangle$ is comparable to that of states $|2\rangle$ and $|1\rangle$ in the optically pumped MOT. To estimate the latter population imbalance, we use stateselective Raman $\pi$ pulses to excite $|2\rangle \rightarrow|3\rangle$ or $|1\rangle \rightarrow|6\rangle$ transitions in the MOT. Probe-induced fluorescence signals from states $|3\rangle$ or $|6\rangle$ show that the initial $|1\rangle$ and $|2\rangle$ populations are equal within $10 \%$. Note that residual population in state $|2\rangle$ is expected to be stable and weakly interacting, since we estimate $\left|a_{32}\right|<30 a_{0}$ for $0 \leq B \leq 50 \mathrm{G}$ using the ABC method, and $a_{12} \simeq 0$ [10]. Using the parameters for the fit shown in Fig. 3, but changing the initial mixture from 50-50 to 60-40, we find a slight increase in the fitted scattering length from $540 a_{0}$ to $563 a_{0}$. Thus, the uncertainty in the calibration of the number of atoms produces the dominant uncertainty and $\left|a_{31}\right|=540_{-100}^{+210} a_{0}$.

To demonstrate that evaporative cooling is occuring, rather than just trap loss, we have also measured the 
final temperature of the mixture using release and recapture [18] from the $\mathrm{CO}_{2}$ laser trap. We obtain $9.8 \pm 1 \mu \mathrm{K}$, which is within $10 \%$ of the final temperature of $8.7 \mu \mathrm{K}$ predicted by the Boltzmann equation model. An excellent fit to the data is obtained for the final temperature, which describes a thermal distribution. However, the initial temperature is not so readily measured, as it is nonthermal before evaporation is initiated, and is rapidly changing during evaporation, unlike the final temperature, which stagnates.

Good fits to the evaporation data are obtained neglecting inelastic collisions, suggesting that the dipolar rate for the $|3\rangle-|1\rangle$ mixture is small, in contrast to the scattering length. A limit on the dipolar loss rate for the $|3\rangle-|1\rangle$ mixture can be estimated from the $\tau=370$ second lifetime of the mixture after evaporation stagnates. For equal populations in both states, dipolar decay results in an initial loss rate $\dot{n}=-G n^{2} / 4$, where $G$ is the dipolar rate constant and $n$ is the total density. To obtain a high density, the trap is loaded at a well depth of $330 \mu \mathrm{K}$ and the temperature of the atoms is reduced by evaporation to $T \simeq 30 \pm 1 \mu \mathrm{K}$. The number of atoms remaining in each state after evaporation is estimated to be $N=6.5 \pm 2.2 \times 10^{4}$, where the uncertainty is in the calibration. We cannot rule out the possibility that one state is depleted on a long time scale, since we do not directly measure the individual state populations. However, we believe that, after evaporation stagnates in the deep well, a $|3\rangle-|1\rangle$ mixture remains, since subsequent reduction of the well depth yields final temperatures consistent with evaporative cooling. Note that the mixture ratio is not critical: An $80-20$ mixture yields an initial loss rate $\dot{n}=-0.16 G n^{2}, \simeq 2 / 3$ that of a $50 / 50$ mixture. For a fixed $330 \mu \mathrm{K}$ trap depth, $\nu^{3}=2.6 \pm 0.3 \mathrm{kHz}^{3}$, and the phase space density for one state in the harmonic approximation is then $\rho_{p h}=N /\left(k_{B} T / h \nu\right)^{3}=7 \times 10^{-4}$. This corresponds to a maximum total density of $n=$ $2 \rho_{p h} / \lambda_{B}^{3}=6.4 \times 10^{11} / \mathrm{cm}^{3}$, where $\lambda_{B} \equiv h / \sqrt{2 \pi M k_{B} T}$. Since the exponential decay time of the $|3\rangle-|1\rangle$ mixture is similar to that obtained in the noninteracting $|1\rangle-|2\rangle$ mixture, we assume the loss is dominated by background gas collisions. Thus, we must have $G n / 4<<1 / \tau$, which yields $G<<2 \times 10^{-14} \mathrm{~cm}^{3} / \mathrm{sec}$. This result is consistent with the value $G \simeq 2 \times 10^{-15} \mathrm{~cm}^{3} / \mathrm{sec}$ predicted for the dipolar rate constant at $30 \mu \mathrm{K}$ by van Abeelen and Verhaar 13.

Future experiments will employ continuous evaporation by slowly reducing the well depth [19]. In this case, very large scattering lengths can be obtained at low temperatures and small well depths by using a reduced bias magnetic field $B$. By adiabatically recompressing the well, experiments can be carried out with the precooled atoms in a deep trap to obtain high density as well. In such experiments, the final low temperature limits the number of atoms in the high energy tail of the energy distribution, exponentially suppressing spin-exchange collisions for $B \neq 0$. For example, if a total of $3 \times 10^{5}$ atoms were contained in our trap at a well depth of $400 \mu \mathrm{K}$, the
Fermi temperature $T_{F}=7 \mu \mathrm{K}$ and the Fermi density is $4 \times 10^{13} / \mathrm{cm}^{3}$. At a temperature of $T=0.1 T_{F}=0.7 \mu \mathrm{K}$, a bias field of $B=0.16 \mathrm{G}$ would split the two-particle hyperfine states by $k_{B} T_{F}+12 k_{B} T$, suppressing the spin exchange rate by $\exp (-12)$, and giving $a_{31} \simeq-1200 a_{0}$. Alternatively, as shown in Fig. 2, large $a_{31}$ can be obtained at moderate $B \simeq 300 \mathrm{G}$.

In conclusion, we have observed that an optically trapped $|3\rangle-|1\rangle$ mixture of ${ }^{6} \mathrm{Li}$ atoms has a very large scattering length at low magnetic field. This mixture is stable against spin-exchange collisions provided that a small bias magnetic field is applied. The evaporation curves measured for this mixture are in good agreement with a model based on an s-wave Boltzmann equation which neglects inelastic processes. We have predicted that the scattering interactions are strongly attractive and widely tunable at low magnetic field. If the parameters described above for deep wells can be attained, the system will be close to the threshold for superfluidity [2] and ideal for investigating frequency shifts and damping in collective oscillations [3,5]. Further, since s-wave interactions can be turned on and off in a few microseconds, this system is well suited for studies of many-body quantum dynamics.

This research has been supported by the Army Research Office and the National Science Foundation.

[1] B. DeMarco, and D. S. Jin, Science 285, 1703 (1999).

[2] H. T. C. Stoof, et al., Phys. Rev. Lett. 76, 10 (1996); See also, M. Houbiers et al., Phys. Rev. A 56, 4864 (1997).

[3] L. Vichi and S. Stringari, Phys. Rev. A 60, 4734 (1999).

[4] R. Combescot, Phys. Rev Lett. 83, 3766 (1999).

[5] G. M. Bruun, and C. W. Clark, Phys. Rev. Lett. 83, 5415 (1999).

[6] G. M. Bruun and C. W. Clark, cond-mat/9906392.

[7] M. Houbiers and H. T. C. Stoof, Phys. Rev. A 59, 1556 (1999).

[8] G. Bruun, et al., Eur. Phys. J. D 7, 433 (1999).

[9] M. Houbiers and H. T. C. Stoof, cond-mat/9808171.

[10] M. Houbiers, et al., Phys. Rev. A 57, R1497 (1998).

[11] E. R. I. Abraham, et al., Phys. Rev A 55, R3299 (1997).

[12] K. M. O'Hara, et al., Phys. Rev. Lett. 82, 4204 (1999).

[13] We are indebted to F. A. van Abeelen and B. J. Verhaar who calculated the inelastic $|\{3,1\}\rangle \rightarrow|\{1,2\}\rangle$ dipolar rate and confirmed our calculations of the magnetic field dependence of $a_{31}$.

[14] P. Törmä and P. Zoller, Phys. Rev. Lett. 85, 487 (2000).

[15] S. Friebel, et al., Phys. Rev. A 57, R20 (1998).

[16] Atomic, Molecular, and Optical Physics Handbook, ed. G. W. Drake, (AIP Press, New York, 1996), p. 176.

[17] O. J. Luiten, et al., Phys. Rev. A 53, 381 (1996).

[18] S. Chu, et al., Phys. Rev. Lett. 55, 48 (1985).

[19] C. S. Adams, et al., Phys. Rev. Lett. 74, 3577 (1995). 